\title{
Face Texture Synthesis from Multiple Images via Sparse and Dense Correspondence
}

\author{
Shugo Yamaguchi ${ }^{1 *}$ \\ Shigeo Morishima ${ }^{2 \dagger}$ \\ ${ }^{1}$ Waseda University \\ ${ }^{2}$ Waseda Research Institute for Science and Engineering
}

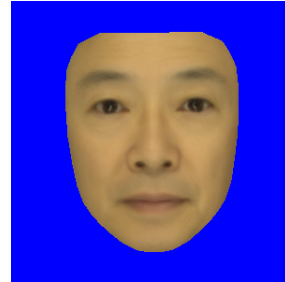

(a) Input Image

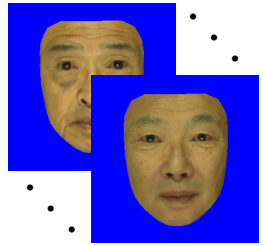

(b) Database
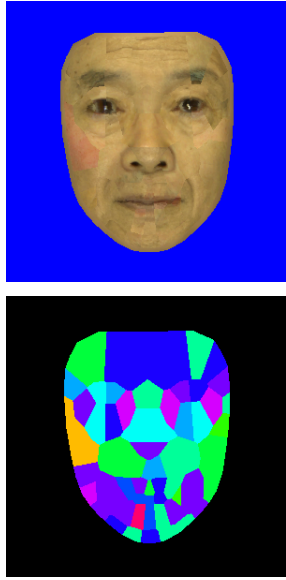

(c) Sparase Matching
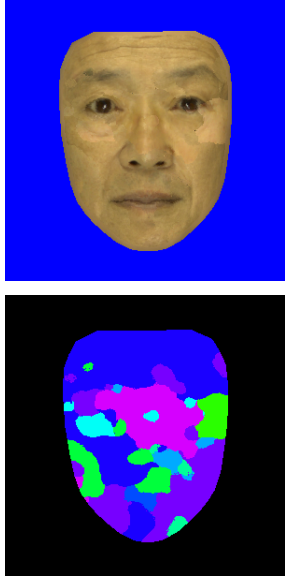

(d) Dense Matching

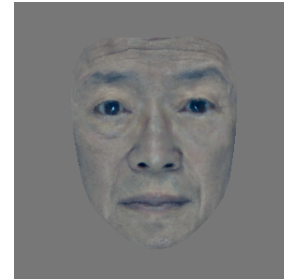

(e) Synthesis by DoG

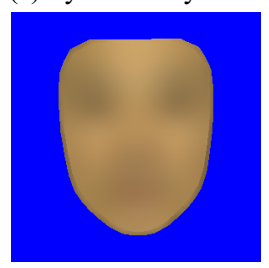

(f) Residual

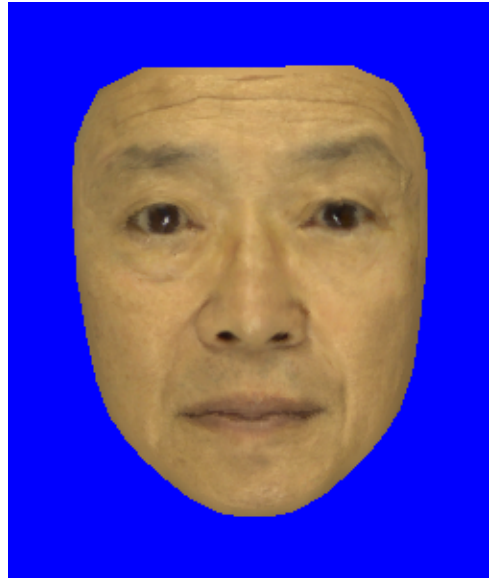

(g) Our Result

Figure 1: Algorithm over view and Average Face example. (a) Input image. This image is average image of (b). (b) Source image database. This example used 60s age male face images. (c) Sparse matching result based on face landmark and KLT tracking. The bottom visualize the corresponding database image indexes. (d) Dense matching by modified PatchMatch algorithm. (e) Optimize the texture by the difference of Gaussian. (f) Residual. The Gaussian blurred input image. ( $g$ ) Our result composed of (e) and (f).

\section{Abstract}

We have a desire to edit images for various purposes such as art, entertainment, and film production so texture synthesis methods have been proposed. Especially, PatchMatch algorithm [Barnes et al. 2009] enabled us to easily use many image editing tools. However, these tools are applied to one image. If we can automatically synthesize from various examples, we can create new and higher quality images. Visio-lization [Mohammed et al. 2009] generated average face by synthesis of face image database. However, the synthesis was applied block-wise so there were artifacts on the result and free form features of source images such as wrinkles could not be preserved. We proposed a new synthesis method for multiple images. We applied sparse and dense nearest neighbor search so that we can preserve both input and source database image features. Our method allows us to create a novel image from a number of examples.

\section{Keywords: Texture synthesis, PatchMatch, Visio-lization}

\section{Concepts: •Computing methodologies $\rightarrow$ Image processing;}

\footnotetext{
*e-mail:wasedayshugo@suou.waseda.jp
}

†e-mail:shigeo@waseda.jp

Permission to make digital or hard copies of all or part of this work for personal or classroom use is granted without fee provided that copies are not made or distributed for profit or commercial advantage and that copies bear this notice and the full citation on the first page. Copyrights for components of this work owned by others than ACM must be honored. Abstracting with credit is permitted. To copy otherwise, or republish, to post on servers or to redistribute to lists, requires prior specific permission and/or a fee. Request permissions from permissions@acm.org. (C) 2016 ACM. SA ’16 Technical Briefs, December 05-08, 2016, Macao

\section{Introduction}

There is a demand for synthesizing a fine facial image including a non-existing human face. Recently, many movies and video games use photo-realistic 3D human model. If we can create new human face textures by little editing and information it may help their productions. Image editing tools such as Adobe Photoshop which uses various algorithms [Barnes et al. 2009], etc. However, these tools can edit a part of an image, but cannot generate a new image.

Visio-lization [Mohammed et al. 2009] synthesized a new facial image from a facial data set. This method divided an input image into grids and optimal database patches were synthesized. This method had problems that the synthesized results were different according to the patch size and could not transfer the free-form feature such as wrinkles.

One of the solutions is to perform dense texture optimization. Visio-lization used Image Quilting [Efros and Freeman 2001] as a texture synthesis method. This method is legacy and rough texture synthesis way. The patch size is large and the patches are sparsely selected and synthesized so the result has artifacts on the border of patches. Texture Optimization [Kwatra et al. 2005] proposed a synthesis method that densely selected patches and optimized texture error. However, it is very time-consuming for using the Texture Optimization method for Visio-lization. It is because Visio-lization checks every database image and select the best patch for each patch. There are some high-performance texture synthesis methods such as PatchMatch [Barnes et al. 2009]. PatchMatch used the local coherence in an image for approximate nearest-neighbor search, but

ISBN: 978-1-4503-4541-5/16/12

DOI: http://dx.doi.org/10.1145/3005358.3005386 


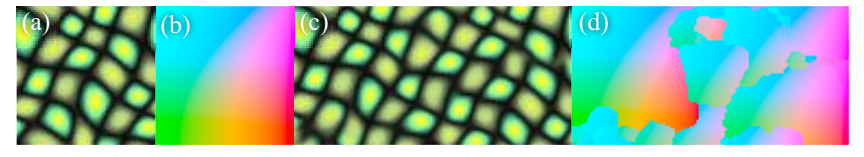

Figure 2: Texture optimization offset map visualizing. (a) Source image. (b) Source image coordinate. (c) Synthesized result. (d) Offset map. The map consists of some clusters.

Visio-lization cannot use this feature because each database image is independent.

Texture optimization method has a feature that when display the nearest-neighbor offset map, the map is constructed by some clusters (Figure 2). Then, when we apply multiple texture synthesis, one cluster should belong to one image. Therefore, a local corresponding image is detected sparsely at first, we should be able to optimize the texture densely.

We propose a new synthesis method. This method

1. sparsely select feature key points

2. based on the key points, initialize the dense correspondence map

3. densely optimize the texture.

This method preserve the input and source images features, and balance the accuracy and the resolution. Our method allows us to create better facial synthesized image for various purposes.

\section{Related Works}

Example-based image synthesis methods with multiple images have been proposed. Visio-lization [Mohammed et al. 2009] could create a new face from a facial database and have some application such as inpainting and smiling. [Risser et al. 2010] created new textures not only face but also denim, skeleton, and butterfly texture based on Visio-lization. [Kawai and Morishima 2015] proposed deblurring method modified Visio-lization. There have been various deblurring methods which do not use the examplebased method but these methods could not apply for other purposes. [Hays et al. 2007] proposed an inpainting method using millions of images, but this method synthesized with only one example selected from database images.

\section{Our Approach}

We synthesize the target image $T$ from the database source image $S_{k}(k \in\{1, \ldots N\}, N$ is the number of database images). We calculate the coordinate $\boldsymbol{y}_{\boldsymbol{k}} \subset S_{k}$ which is corresponding to $\boldsymbol{x} \subset T$. Here, we define the offset $\boldsymbol{v}_{k}$,

$$
\boldsymbol{y}_{\boldsymbol{k}}=\boldsymbol{x}+\boldsymbol{v}_{k}(\boldsymbol{x})
$$

and determine it by the following algorithm.

\subsection{Sparse Correspondence}

We first take sparse feature points. When we treat face problem, we can use the face landmarks. Now we take $n$ landmarks $p_{0 j}(j \in\{1, \ldots, n\})$ from input face image using [Kazemi and Sullivan 2014] and get the landmarks of the database images $p_{k j}$.

We get the initial offset $\boldsymbol{v}_{r b f}(\boldsymbol{x})$ from the landmarks by Radial Basis Function (RBF) interpolation.
Next to that, we match the feature points between $T$ and $S_{k}$ by KLT tracking algorithm [Tomasi and Kanade 1991]. We define $p_{k j}$ as the initial guess and track the point $p_{k j}^{\prime}=f_{k l t}\left(p_{0 j}, p_{k j}\right)$. We define the offfset $\boldsymbol{v}_{k j}^{\prime}(\boldsymbol{x})$ from initial RBF as

$$
\boldsymbol{v}_{k j}^{\prime}(\boldsymbol{x})=p_{k j}^{\prime}-p_{k j}
$$

where

$$
\begin{gathered}
k(j)=\arg \min e r r_{k l t}\left(p_{k j}^{\prime}, p_{0 j}\right) \\
j(\boldsymbol{x})=\arg \min _{j}\left\|\boldsymbol{x}-p_{0 j}\right\|
\end{gathered}
$$

It means that the input image is divided by Voronoi cells and assigned the area of the locally corresponding database image. We can get the initial sparse offset map

$$
\boldsymbol{v}_{k}(\boldsymbol{x})=\boldsymbol{v}_{r b f}(\boldsymbol{x}, k)+\boldsymbol{v}_{k j}^{\prime}(\boldsymbol{x}) .
$$

\subsection{Dense Correspondence}

We calculate the approximate nearest neighbor patches. We modify the PatchMatch Algorithm [Barnes et al. 2009] so that we can use multiple source images. PatchMatch Algorithm is composed of propagation and random search steps. When we update offset $v$ at each step, we also update the database number $k$.

Propagation. We compare the current patch with the left patch and the upper patch. That is, we select

$$
\min \left[D\left(\boldsymbol{x}_{0}, \boldsymbol{y}_{0}\right), D\left(\boldsymbol{x}_{1}, \boldsymbol{y}_{1}\right), D\left(\boldsymbol{x}_{2}, \boldsymbol{y}_{2}\right)\right]
$$

where $\boldsymbol{x}_{0}=(x, y), \boldsymbol{x}_{1}=(x-1, y), \boldsymbol{x}_{2}=(x, y-1)$, and $\boldsymbol{y}_{i}=$ $\boldsymbol{y}_{k}\left(\boldsymbol{x}_{i}\right) . D(\boldsymbol{x}, \boldsymbol{y})$ is the distance function between the two patches.

$$
D\left(\boldsymbol{x}, \boldsymbol{y}_{k}\right)=D_{0}\left(\boldsymbol{x}, \boldsymbol{y}_{k}\right)+\lambda\left\|\boldsymbol{v}(\boldsymbol{x}, k)-\boldsymbol{v}_{r b f}(\boldsymbol{x}, k)\right\|
$$

$D_{0}$ is the sum of squared distance of the target patch $P(\boldsymbol{x})$ and the source patch $Q\left(\boldsymbol{y}_{k}\right)$. Here, we take from the difference of gaussian $L$ so that we can reduce the effect of the lighting environment or the skin color inspired by [Shih et al. 2014].

$$
L(I)=I-I * G\left(2^{4}\right)
$$

where $I$ is the original image of $T$ or $S_{k}, G(\sigma)$ is the Gaussian kernel. We define the residual as:

$$
R(I)=I * G\left(2^{4}\right)
$$

In addition to it, we consider the constraint against the offset map $\boldsymbol{v}(\boldsymbol{x}, k)$. We can preserve the geometry of the face by this constraint. We define $\lambda=1.0$.

Random Search. We randomly search around the current offset. In this step, we also update $k$.

$$
\boldsymbol{u}_{i k^{\prime}}=\boldsymbol{v}_{k}+w \alpha^{i} \boldsymbol{R}_{i}
$$

where $\boldsymbol{R}_{i}$ is a uniform random in [-1,1] $\times[-1,1], w$ is the max search window size, and $\alpha$ is a fixed ratio between search window sizes. We examine patches for $\mathrm{i}=0,1,2, \ldots$ until the current search radius $w \alpha^{i}$ is below 1 pixel. In our applications $w=15, \alpha=0.5$. 


\subsection{Texture Synthesis}

In this section, we synthesize the texture. Visio-lization erased the discontinuity on the border by Poisson Image Editing (PIE) Algorithm [Pérez et al. 2003]. However, this algorithm is not effective if the synthesis area is small or complicated. It is because PIE synthesis gradient based on the border condition. If the border is blurred and the area is small, we cannot ignore the border blur because the border value is preserved. Therefore, we synthesize the image by using the difference of Gaussian $L(I)$ and optimizing the distance measure

$$
d(L(T), L(S))=\frac{1}{N_{T}} \sum_{\boldsymbol{x} \subset L(T)} \min _{\boldsymbol{y}_{k} \subset L\left(S_{k}\right)} D\left(\boldsymbol{x}, \boldsymbol{y}_{k}\right) .
$$

$d(T, S)$ is minimized by the EM-like algorithm. We iterate the approximate nearest-neighbor search and synthesis of the texture. Target texture $L(T)$ is calculated by the following equation to optimize the $d(L(T), L(S))$

$$
L(T(\boldsymbol{x}))=\frac{1}{N_{T}} \sum_{T}^{N} L\left(S_{k}\left(\boldsymbol{y}_{k}\right)\right) .
$$

Finally, we get the result Dst by synthesizing the difference of Gaussian and the residual of the input image.

$$
D s t=L(T)+R(T) \text {. }
$$

We optimize the texture by iterating 3.2 and 3.3 steps.

\section{Applications and Results}

We have implemented our method in $\mathrm{C}++$. It usually takes around 3 seconds to synthesize one $300 \times 300$ pixels on a $2.3 \mathrm{GHz} \mathrm{CPU}$ with 1 cores. The dominant time-consuming step is 3.2 Dense Correspondence. We search by 2 iterations and optimize image by 3 iterations. We use patch size 17, 9, 5 for each optimizing step.

\subsection{Average Face}

Simple average face composed of multiple images is blurred because each image cancels the other feature. We input a simple average image and reconstruct it from the original source images by our method. We show average face reconstruction results in Figure 3. Previous work's correspondence map was block-wise so it could not preserve source image features such as long wrinkle. Our method preserves both source image features and input image local shapes.

\subsection{Deblurrering}

We show deblurring results and comparison in Figure 4.

In order to evaluate the qualities, we define Error and Coarseness. Error $E(I)$ is the Root Mean Square Error (RMSE) between ground truth and result in $\mathrm{L}^{*} \mathrm{a} * \mathrm{~b} *$ color space.

$$
E(I)=\sqrt{\frac{1}{N} \sum_{x}^{N}\left\|I(x)-I_{g t}(x)\right\|^{2}}
$$

where $\mathrm{I}$ is the result image, $I(x)$ is pixel value at a coordinate $x$ in $I, I_{g t}$ is GroundTruth, $N$ is the number of pixels. In order to define Coarseness, we define Fineness as:

$$
F(I)=\sqrt{\frac{1}{N} \sum_{x}^{N}\|L(x)\|^{2}}
$$
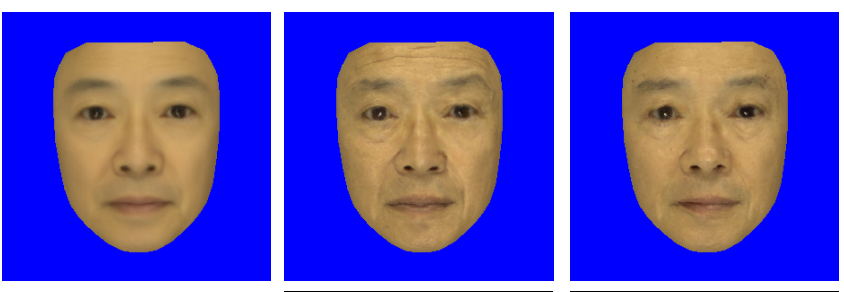

Input Image

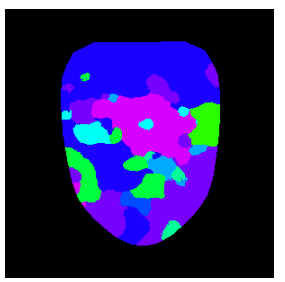

Our Result

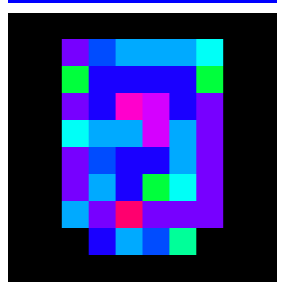

[Mohammed et al. 2009] Patch size: $31 \times 31$
Figure 3: Average Face results. The input image is composed of 60 s age male face images. The number of images is 16. Previous work's correspondence map was block-wise. Our method's correspondence map is optimized and preserve the source image features such as wrinkle.

where $L$ is defined as:

$$
L_{l}[I]=I-I * G\left(2^{l}\right)
$$

where $G(\sigma)$ is Gaussian kernel, we use $l=1$. Figure 5 is comparison of $F_{l}$ values between different resolution images. It shows fine resolution image has high $L_{0}$ value and coarse resolution has low value. Using Fineness, we can define Coarseness as:

$$
C(I)=\frac{F\left(I_{g t}\right)}{F(I)}
$$

Figure 6 shows the comparison of Error-Coarseness graph. In [Mohammed et al. 2009], they were trade-off values. If the patch size was large, Coarseness was low but RMSE was high. If the patch size was small, RMSE was low but Coarseness was high. Figure 4 and 6 shows that our result improve the balance between Error and Coarseness.

\section{Conclusion and Future works}

We proposed a new synthesis method for multiple images. We applied sparse and dense nearest neighbor search so that we can preserve both input and source database image features. Our method allows us to create a novel image from a number of examples.

Since face is the most suitable target for the multiple texture synthesis, we applied this method only to faces in this paper. Our method will be able to improve not only facial synthesis but also general multiple texture synthesis. As a future work, we want to apply this method to general examples.

\section{Acknowledgements}

We thank Keisuke Ogaki for providing valuable comments on resolution evaluation. We also thank Tsukasa Fukusato for providing valuable comments on this paper.

\section{References}

Barnes, C., Shechtman, E., Finkelstein, A., And GoldMAN, D. B. 2009. PatchMatch: A Randomized Correspondence 

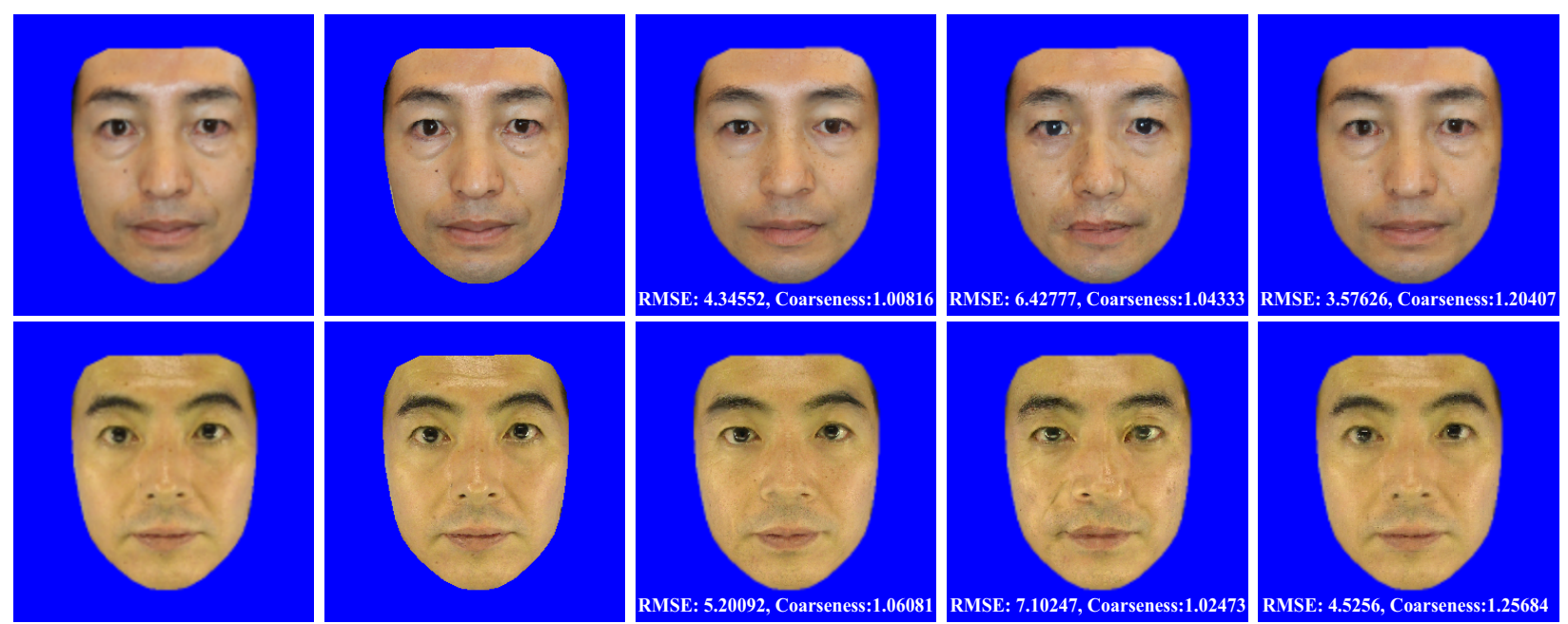

Input Image

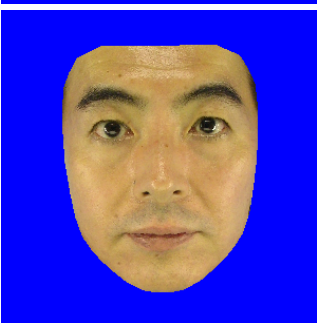

Ground Truth

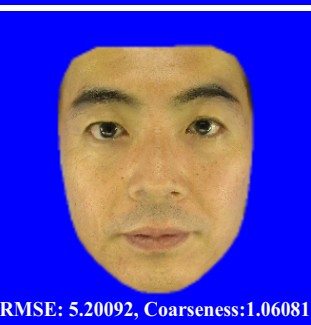

Our Result

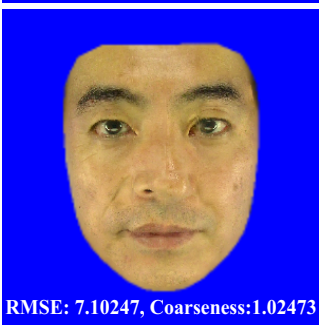

[Mohammed et al. 2009]

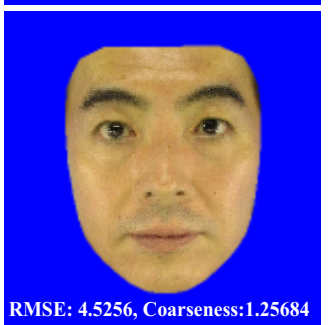

Patch size: $5 \times 5$

Figure 4: Deblurring results. RMSE and Coarseness were trade-off in previous works. Our result meats both accuracy and high resolution.

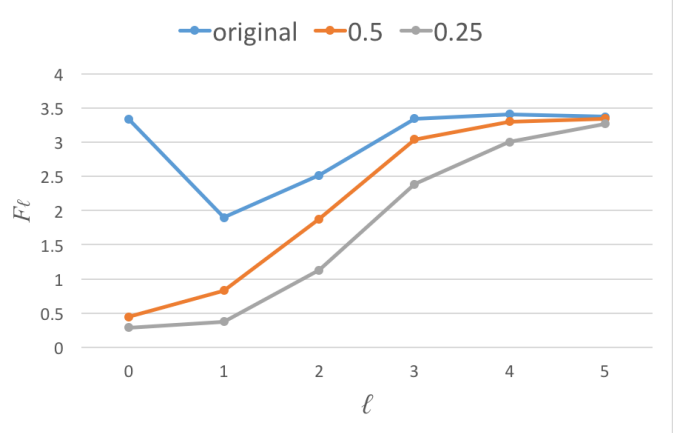

Figure 5: Comparison of $F_{l}$ values between different resolution images. We compare the original image, down-sampled image in 0.5 scales and 0.25 scales. Down-sampled image are resized as original image size by bi-linear interpolation.

Algorithm for Structural Image Editing. ACM Transactions on Graphics 28, 3, 1.

Efros, A. A., AND Freeman, W. T. 2001. Image Quilting for Texture Synthesis and Transfer. Siggraph, August, 1-6.

Hays, J., Efros, A. A., Hays, J., And Efros, A. A. 2007. Scene completion using millions of photographs. In ACM SIGGRAPH 2007 papers on - SIGGRAPH '07, ACM Press, New York, New York, USA, vol. 26, 4.

Kawai, M., And Morishima, S. 2015. FOCUSING PATCH: Automatic Photorealistic Deblurring for Facial Images by PatchBased Color Transfer. Springer International Publishing, 155166.

Kazemi, V., And Sullivan, J. 2014. One millisecond face alignment with an ensemble of regression trees. In Proceedings of the IEEE Conference on Computer Vision and Pattern Recognition, 1867-1874.

Kwatra, V., Essa, I., Bobick, A., And Kwatra, N. 2005. Texture optimization for example-based synthesis. ACM Transactions on Graphics 24, 3, 795.

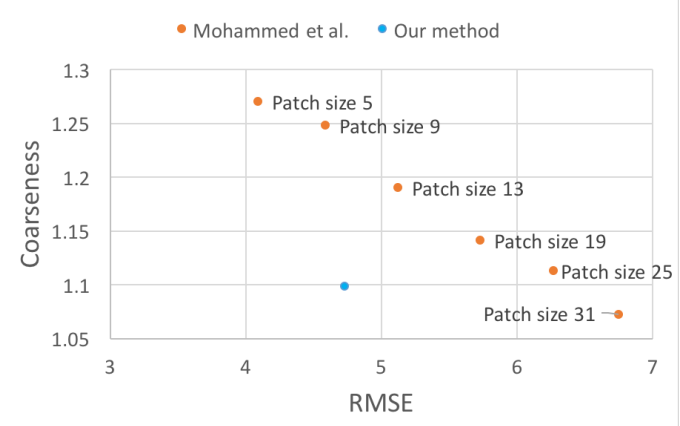

Figure 6: Error-Coarseness score comparison between our method and previous work. Each dot is the average score of each method and patch size. Our method improves the balance between error and coarseness.

Mohammed, U., Prince, S., And Kautz, J. 2009. Visiolization: generating novel facial images. ACM Transactions on Graphics 28, 3, 1-8.

Pérez, P., Gangnet, M., and Blake, A. 2003. Poisson image editing. In ACM Transactions on Graphics (TOG), vol. 22, ACM, 313-318.

Risser, E., HAN, C., DAHYOT, R., AND GRINSPUn, E. 2010. Synthesizing structured image hybrids. ACM Transactions on Graphics 29, 4, 1.

Shih, Y., Paris, S., Barnes, C., Freeman, W. T., and DuRAND, F. 2014. Style transfer for headshot portraits. MIT web domain.

Tomasi, C., And Kanade, T. 1991. Detection and tracking of point features. School of Computer Science, Carnegie Mellon Univ. Pittsburgh. 